\title{
Systolic blood pressure is superior to other haemodynamic predictors of outcome in community acquired pneumonia
}

\author{
J D Chalmers, A Singanayagam, A T Hill
}

\section{See Editorial, p 665}

Royal Infirmary of Edinburgh, Edinburgh, UK

Correspondence to: Dr J Chalmers, Department of Respiratory Medicine, Royal Infirmary of Edinburgh, 51 Little France Crescent, Old Dalkeith Road, Edinburgh EH16 4SA, UK; jamesdchalmers@ googlemail.com

Received 7 January 2008 Accepted 23 April 2008 Published Online First 20 May 2008

\begin{abstract}
Introduction: Admission blood pressure (BP) assessment is a central component of severity assessment for community acquired pneumonia. The aim of this study was to establish which readily available haemodynamic measure on admission is most useful for predicting severity in patients admitted with community acquired pneumonia.
\end{abstract}

Methods: A prospective observational study of patients admitted with community acquired pneumonia was conducted in Edinburgh, UK. The measurements compared were systolic and diastolic BP, mean arterial pressure and pulse pressure. The outcomes of interest were 30 day mortality and the requirement for mechanical ventilation and/or inotropic support.

Results: Admission systolic BP $<90 \mathrm{~mm} \mathrm{Hg}$, diastolic BP $\leqslant 60 \mathrm{~mm} \mathrm{Hg}$, mean arterial pressure $<70 \mathrm{~mm} \mathrm{Hg}$ and pulse pressure $\leqslant 40 \mathrm{~mm} \mathrm{Hg}$ were all associated with increased 30 day mortality and the need for mechanical ventilation and/or inotropic support on multivariate logistic regression. The AUC values for each predictor of 30 day

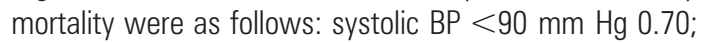

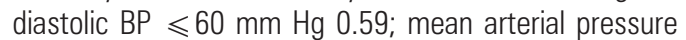
$<70 \mathrm{~mm} \mathrm{Hg} \mathrm{0.64}$; and pulse pressure $\leqslant 40 \mathrm{~mm} \mathrm{Hg}$ 0.60 . The AUC values for each predictor of need for mechanical ventilation and/or inotropic support were as follows: systolic BP $<90 \mathrm{~mm} \mathrm{Hg} \mathrm{0.70;} \mathrm{diastolic} \mathrm{BP}$ $\leqslant 60 \mathrm{~mm} \mathrm{Hg} \mathrm{0.68}$; mean arterial pressure $<70 \mathrm{~mm} \mathrm{Hg}$ 0.69 ; and pulse pressure $\leqslant 40 \mathrm{~mm} \mathrm{Hg} \mathrm{0.59}$. A simplified CRB65 score containing systolic blood pressure $<90 \mathrm{~mm} \mathrm{Hg}$ alone performed equally well to standard CRB65 score (AUC 0.76 vs 0.74 ) and to the standard CURB65 score (0.76 vs 0.76 ) for the prediction of 30 day mortality. The simplified CRB65 score was equivalent for prediction of mechanical ventilation and/or inotropic support to standard CRB65 (0.77 vs 0.77) and to CURB65 (0.77 vs 0.78 ).

Conclusion: Systolic BP is superior to other haemodynamic predictors of 30 day mortality and need for mechanical ventilation and/or inotropic support in community acquired pneumonia. The CURB65 score can be simplified to a modified CRB65 score by omission of the diastolic BP criterion without compromising its accuracy.

Severity assessment rules are increasingly promoted for use in a variety of disorders, including community acquired pneumonia. Hypotension is the defining feature of sepsis and sepsis shock ${ }^{1}$ and has been consistently found to be associated with increased mortality in patients with community acquired pneumonia. ${ }^{2-5}$

Different severity rules recommend different blood pressure measurements. The Pneumonia
Severity Index is the most widely used pneumonia severity score worldwide and recommends systolic blood pressure $<90 \mathrm{~mm} \mathrm{Hg}$ as one its core high risk features. ${ }^{3}$ In the UK, the CURB65 and CRB65 prediction scores have been in use since 2004 following a refinement of previous British Thoracic Society rules. The CURB65 score recommends either systolic blood pressure $<90 \mathrm{~mm} \mathrm{Hg}$ or diastolic blood pressure $\leqslant 60 \mathrm{~mm} \mathrm{Hg}$. ${ }^{67}$ The APACHE II score is the most widely used generic scoring system for predicting mortality in acutely ill patients, particularly in the intensive care unit, and uses mean arterial pressure as the preferred haemodynamic measure. ${ }^{8}$ Systolic blood pressure is used in the Modified Early Warning Score which is widely used for acute medical and surgical admissions in UK hospitals. ${ }^{9}$ Pulse pressure has not previously been considered as a predictor of outcome in community acquired pneumonia, although it is a readily available clinical measure and animal models have shown a rapid increase in pulse pressure at onset of sepsis. ${ }^{10}$ The original British Thoracic Society study showed that low diastolic blood pressure was associated with mortality, independent of systolic blood pressure. ${ }^{2}$ A low diastolic pressure with a normal systolic blood pressure leads to an elevated pulse pressure. The authors therefore hypothesised that an elevated pulse pressure would be associated with a poor outcome.

In this study, we investigated which readily available admission blood pressure measurement was most accurate at predicting the two most important outcomes in community acquired pneumonia-30 day mortality and need for invasive ventilation and/or inotropic support. In addition, we sought to establish if modifying the CURB65 score using different haemodynamic measurements was of additional value.

\section{METHODS}

A prospective observational study of consecutive unselected patients admitted to NHS Lothian University Hospitals Division (Edinburgh, UK) between January 2005 and November 2007 with a diagnosis of community acquired pneumonia was carried out. The study was approved by the Lothian Research Ethics Committee.

Community acquired pneumonia was diagnosed using the following criteria recommended by the British Thoracic Society guidelines: ${ }^{11}$

- symptoms and signs consistent with an acute lower respiratory tract infection associated with new radiographic shadowing for which there is no other explanation; 
Table 1 Baseline characteristics of the study population

\begin{tabular}{lc}
\hline Baseline characteristic & $\begin{array}{l}\text { Study population } \\
\text { (n= 1007) }\end{array}$ \\
\hline Age (y) & $66(50-78)$ \\
Gender (\% male) & 49.7 \\
Duration of admission (days) & $5(3-11)$ \\
Chronic cardiac failure (\%) & 20 \\
Cerebrovascular disease (\%) & 11.6 \\
Chronic renal failure (\%) & 6 \\
Diabetes mellitus (\%) & 10.1 \\
COPD (\%) & 20.6 \\
Other chronic lung diseases (\%) & 3.6 \\
Current smokers (\%) & 34.2 \\
Ex-smokers (\%) & 21.4 \\
Non-smokers (\%) & 44.4 \\
\hline
\end{tabular}

COPD, chronic obstructive pulmonary disease.

- the illness is the primary reason for hospital admission and is managed as pneumonia.

In practice, patients were included in the study if they presented with a new infiltrate on a chest radiograph and had three or more of the following symptoms or signs: cough, sputum production, breathlessness, pleuritic chest pain, haemoptysis, fever, headache, signs consistent with pneumonia on chest auscultation.

Exclusion criteria were: hospital acquired pneumonia (development of symptoms $>48 \mathrm{~h}$ following admission or discharge from an acute care facility $<2$ weeks prior to admission); active malignancy; immunosuppression; pulmonary embolism; and patients in whom active treatment was not considered appropriate (palliative care).

\section{Site of care}

At the study sites, patients present either as self-referral to accident and emergency or via general practitioner referral to the medical assessment unit. At both sites, patients are reviewed by the medical team and the decision to admit or discharge the

Table 2 Blood pressure measurements and 30 day mortality or need for mechanical ventilation and/or inotropic support using different cut-off values

\begin{tabular}{lll}
\hline & $\begin{array}{l}\text { 30 day } \\
\text { mortality (\%) }\end{array}$ & $\begin{array}{l}\text { Need for mechanical } \\
\text { ventilation and/or } \\
\text { inotropic support (\%) }\end{array}$ \\
\hline Systolic BP (mm Hg) & & \\
$0-60$ & $40^{* * *}$ & $73.3^{* * *}$ \\
$61-89$ & 29.5 & 30.4 \\
$90+$ & 6.7 & 6.7 \\
Diastolic BP (mm Hg) & & \\
$0-39$ & $28.6^{* * *}$ & $57.1^{* * *}$ \\
$41-60$ & 13.3 & 16.0 \\
$61+$ & 7.2 & 5.7 \\
MAP (mm Hg) & & \\
$0-49$ & $40^{* * *}$ & $60^{* * *}$ \\
$50-69$ & 21.5 & 24.9 \\
$70+$ & 7.1 & 5.2 \\
Pulse pressure (mm Hg) & & \\
$<30$ & $20^{* * *}$ & $23.3^{* * *}$ \\
$31-40$ & 15.6 & 17.8 \\
$41-60$ & 7.5 & 6.1 \\
$61-80$ & 7.1 & 10.2 \\
$80+$ & 9.0 & 7.7 \\
\hline
\end{tabular}

***Kruskal-Wallis test, $\mathrm{p}<0.001$

$\mathrm{BP}$, blood pressure; MAP, mean arterial pressure. patient is made. Subsequently, patients spend $12-24 \mathrm{~h}$ in the medical assessment unit from where they may be discharged or move on to a specialist ward. Critically ill patients may be admitted at any time to the intensive care unit for invasive ventilation or inotropic support or to a high dependency unit which provides intensive monitoring as well as non-invasive ventilation (bi-level or continuous positive airways pressure ventilation) and/or inotropic support. Patients were enrolled in the study from both accident and emergency departments and the medical assessment units.

\section{Study protocol}

For all patients admitted with community acquired pneumonia, a proforma was completed on admission that included patient observations (blood pressure, pulse, respiratory rate, temperature), and standard blood tests were obtained for each patient (full blood count, urea and electrolytes, liver function tests, coagulation profile and $\mathrm{C}$ reactive protein). All observations were taken in the emergency department within $4 \mathrm{~h}$ of arrival. On admission, patients were risk assessed using the British Thoracic Society CURB65 score, CRB65 score and the Pneumonia Severity Index. All patients received standard antibiotic therapy in accordance with British Thoracic Society guidelines. ${ }^{11}$

\section{Blood pressure measurements}

Blood pressure measurements (systolic and diastolic) were made within $4 \mathrm{~h}$ of admission in all patients. Measurements were taken prior to intravenous fluid resuscitation or inotropic support except in patients who had received paramedic resuscitation prior to hospital admission. Measurements were made electronically. Four blood pressure measurements were readily available at admission-systolic, diastolic, mean arterial pressure and pulse pressure. Pulse pressure is defined as systolic blood pressure - diastolic blood pressure. Mean arterial pressure was calculated as diastolic blood pressure $+1 / 3 \times$ pulse pressure.

Cut-offs for systolic, diastolic and mean arterial pressure were selected for analysis based on the available literature and cutoffs used in previous studies of severity scores. From previous studies, systolic blood pressure $<90 \mathrm{~mm} \mathrm{Hg}$, diastolic blood pressure $\leqslant 60 \mathrm{~mm} \mathrm{Hg}$ and mean arterial pressure $<70 \mathrm{~mm} \mathrm{Hg}$ are the most consistently used to define increased risk. Pulse pressure has not been studied previously. The authors considered a pulse pressure of $40 \mathrm{~mm} \mathrm{Hg}$ as normal.

\section{Outcomes}

The primary outcome of interest was 30 day mortality and requirement for mechanical ventilation and/or inotropic support.

\section{Statistical analysis}

All data were analysed using SPSS V.13 for windows. Descriptive statistics of demographic and clinical variables are presented as median (IOR) unless otherwise stated. The

Table 3 Haemodynamic predictors of 30 day mortality in community acquired pneumonia

\begin{tabular}{lllc}
\hline Predictor & n & OR (CI) & p Value \\
\hline Systolic blood pressure $<90 \mathrm{~mm} \mathrm{Hg}$ & 187 & $5.6(3.4$ to 9.3$)$ & $<0.0001$ \\
Diastolic blood pressure $\leqslant 60 \mathrm{~mm} \mathrm{Hg}$ & 282 & $1.9(1.2$ to 3.0$)$ & 0.006 \\
Mean arterial pressure $<70 \mathrm{~mm} \mathrm{Hg}$ & 259 & $3.1(2.0$ to 5.0$)$ & $<0.0001$ \\
Pulse pressure $\leqslant 40 \mathrm{~mm} \mathrm{Hg}$ & 240 & $1.9(1.2$ to 3.1$)$ & 0.005 \\
\hline
\end{tabular}


Table 4 Haemodynamic predictors of need for mechanical ventilation and/or inotropic support in community acquired pneumonia

\begin{tabular}{lllc}
\hline Predictor & n & OR (CI) & p Value \\
\hline Systolic blood pressure $<90 \mathrm{~mm} \mathrm{Hg}$ & 187 & $5.6(3.4-9.4)$ & $<0.0001$ \\
Diastolic blood pressure $\leqslant 60 \mathrm{~mm} \mathrm{Hg}$ & 282 & $2.9(1.8-4.7)$ & 0.0001 \\
Mean arterial pressure $<70 \mathrm{~mm} \mathrm{Hg}$ & 259 & $4.6(2.9-7.3)$ & $<0.0001$ \\
Pulse pressure $\leqslant 40 \mathrm{~mm} \mathrm{Hg}$ & 240 & $1.7(1.1-2.6)$ & 0.02 \\
\hline
\end{tabular}

Kruskal-Wallis test was used for comparison of more than two groups. The authors used multiple logistic regression to establish if each haemodynamic variable was associated with the outcomes. Continuous variables were converted to binary based on cut-off points established in the literature as predictive of high risk. To the baseline model we included age and pneumonia severity (pneumonia severity index score), gender, comorbidity (chronic cardiac failure, cerebrovascular disease, chronic renal failure, chronic obstructive pulmonary disease, diabetes mellitus) and smoking status coded as binary variables.

The value of tests for predicting outcomes was compared using the area under the receiver operator characteristic curve (AUC). For interpretation of these values the following is widely accepted ${ }^{12}$ : AUC $0.50-0.59=$ no value of test; $0.60-0.69$ $=$ poor discriminatory value; $0.70-0.79=$ moderate discriminatory value; $0.80-0.89=$ good discriminatory value; $0.90-1.00$ $=$ excellent discriminatory value. A $\mathrm{p}$ value of $<0.05$ was considered statistically significant for each analysis.

\section{RESULTS}

A total of 1007 patients were included in the study. Baseline characteristics are shown in table 1 . We found that $14.1 \%$ of patients were discharged within $24 \mathrm{~h}$ of admission. The 30 day mortality rate was $9.6 \%$ and $10.2 \%$ of patients required invasive ventilation and/or inotropic support. Of those patients that died, $47.4 \%$ had a do not attempt resuscitation order or did not receive escalation of treatment beyond ward management.

\section{Haemodynamic predictors of mortality in community acquired pneumonia}

The association between systolic, diastolic, mean arterial blood pressure and pulse pressure at different cut-points with 30 day mortality and need for mechanical ventilation and/or inotropic support were compared (table 2). With reducing systolic blood pressure, diastolic blood pressure, mean arterial pressure and pulse pressure there was increasing 30 day mortality and need for mechanical ventilation and/or inotropic support.

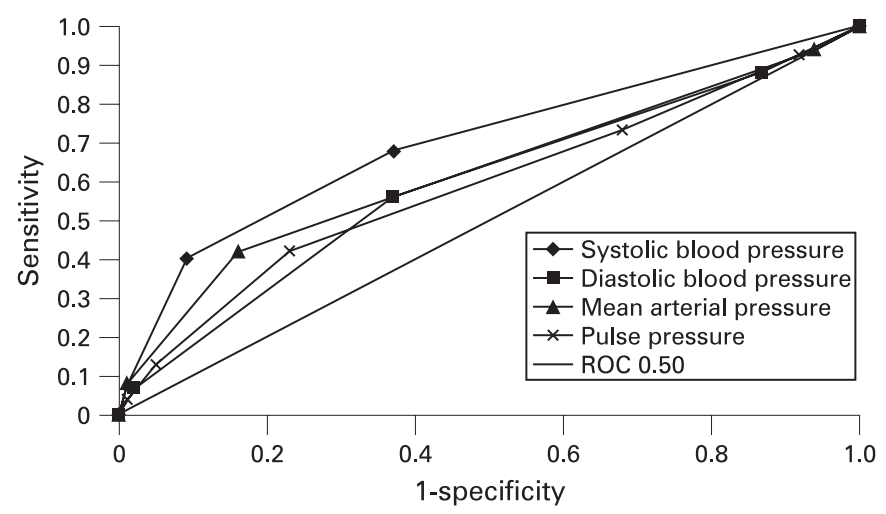

Figure 1 Haemodynamic variables and prediction of 30 day mortality. ROC, receiver operator characteristic curve.

We hypothesised that increased pulse pressure would be associated with poor outcome. This was disproved however as an increased pulse pressure $>40 \mathrm{~mm} \mathrm{Hg}$ was not associated with increased 30 day mortality or need for mechanical ventilation and/or inotropic support.

On multivariate logistic regression, reduced systolic blood pressure $<90 \mathrm{~mm} \mathrm{Hg}$, diastolic blood pressure $\leqslant 60 \mathrm{~mm} \mathrm{Hg}$, mean arterial pressure $<70 \mathrm{~mm} \mathrm{Hg}$ and pulse pressure $\leqslant 40 \mathrm{~mm} \mathrm{Hg}$ were identified as independent predictors of 30 day mortality (table 3 ) and mechanical ventilation and/or inotropic support (table 4).

Comparison of haemodynamic variables for prediction of $\mathbf{3 0}$ day mortality and mechanical ventilation and/or inotropic support

Each haemodynamic variable was compared for its ability to predict 30 day mortality and need for mechanical ventilation and/or inotropic support (table 5). Systolic blood pressure had the highest area under the curve for each of the outcomes considered.

Receiver operator characteristic curves are shown for each haemodynamic variable and prediction of 30 day mortality (fig 1) and need for mechanical ventilation and/or inotropic support (fig 2).

\section{Systolic and diastolic blood pressure measurements and the CURB65 score}

Ninety-five patients (33.7\%) with diastolic hypotension ( $\leqslant 60 \mathrm{mmHg}$ ) did not have accompanying systolic hypotension. Multivariate logistic regression was performed comparing patients with diastolic hypotension (diastolic blood pressure

Table 5 Predictive value of haemodynamic measurements in community acquired pneumonia

\begin{tabular}{|c|c|c|c|c|c|c|}
\hline & $\begin{array}{l}\text { PPV } \\
(\%)\end{array}$ & $\begin{array}{l}\text { NPV } \\
(\%)\end{array}$ & $\begin{array}{l}\text { Sensitivity } \\
\text { (\%) }\end{array}$ & $\begin{array}{l}\text { Specificity } \\
(\%)\end{array}$ & AUC & p Value \\
\hline \multicolumn{7}{|l|}{ Prediction of 30 day mortality } \\
\hline Systolic blood pressure $<90 \mathrm{~mm} \mathrm{Hg}$ & 30.7 & 93.3 & 41.1 & 89.9 & $0.70(0.67-0.74)$ & $<0.0001$ \\
\hline Diastolic blood pressure $\leqslant 60 \mathrm{~mm} \mathrm{Hg}$ & 14.2 & 92.8 & 53.7 & 64.7 & $0.59(0.56-0.62)$ & 0.006 \\
\hline Mean arterial pressure $<70 \mathrm{~mm} \mathrm{Hg}$ & 18.3 & 92.9 & 43.3 & 79.5 & $0.64(0.61-0.67)$ & 0.001 \\
\hline Pulse pressure $\leqslant 40 \mathrm{mmHg}$ & 14.2 & 93.0 & 53.6 & 64.4 & $0.60(0.56-0.63)$ & 0.003 \\
\hline \multicolumn{7}{|c|}{ Prediction of need for mechanical ventilation and/or inotropic support } \\
\hline Systolic blood pressure $<90 \mathrm{~mm} \mathrm{Hg}$ & 35.4 & 93.3 & 44.6 & 90.5 & $0.70(0.67-0.73)$ & $<0.0001$ \\
\hline Diastolic blood pressure $\leqslant 60 \mathrm{~mm} \mathrm{Hg}$ & 18.4 & 94.3 & 65.3 & 66.2 & $0.68(0.65-0.72)$ & $<0.0001$ \\
\hline Mean arterial pressure $<70 \mathrm{~mm} \mathrm{Hg}$ & 24.5 & 94.8 & 57.7 & 81.3 & $0.69(0.66-0.72)$ & $<0.0001$ \\
\hline Pulse pressure $\leqslant 40 \mathrm{~mm} \mathrm{Hg}$ & 15.3 & 92.7 & 54.4 & 65.6 & $0.59(0.56-0.63)$ & 0.002 \\
\hline
\end{tabular}




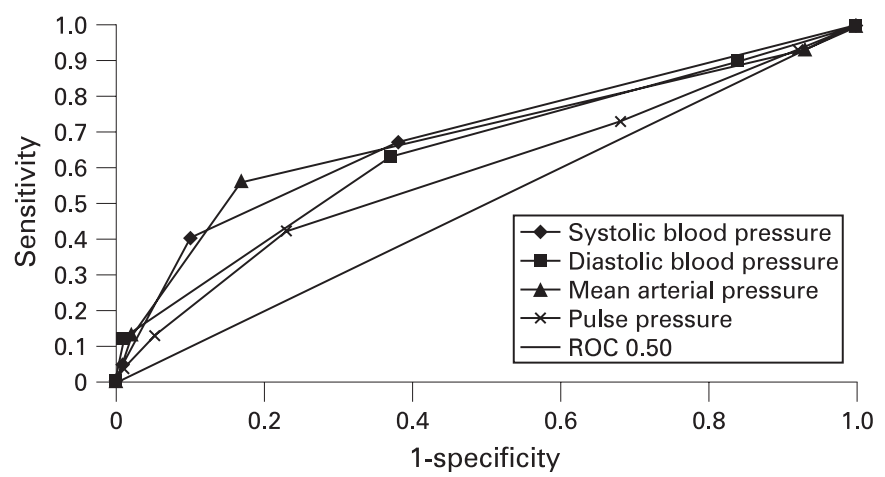

Figure 2 Haemodynamic variables and prediction of need for mechanical ventilation and/or inotropic support. ROC, receiver operator characteristic curve.

$\leqslant 60 \mathrm{~mm} \mathrm{Hg}$ ) in the absence of systolic hypotension (systolic blood pressure $\geqslant 90 \mathrm{~mm} \mathrm{Hg}$ ). Diastolic hypotension in the absence of systolic hypotension was not associated with increased 30 day mortality (odds ratio 1.4 (0.7 to 2.8); $p=0.3$ ) or need for mechanical ventilation and/or inotropic support (odds ratio $0.8(0.4-1.3) ; \mathrm{p}=0.4)$.

\section{Modification of the CURB65 and CRB65 scores}

The performance of simplified CURB65 and CRB65 scores using systolic blood pressure $<90 \mathrm{~mm} \mathrm{Hg}$ alone were compared with standard CURB65 and CRB65 scores (table 6). Combining systolic and diastolic blood pressure has no additional benefit over using systolic blood pressure alone.

\section{DISCUSSION}

This study has demonstrated that admission blood pressure can predict 30 day mortality and need for mechanical ventilation and/or inotropic support in patients admitted with community acquired pneumonia. Systolic blood pressure is superior to other haemodynamic measures in predicting 30 day mortality and the need for mechanical ventilation and/or inotropic support. In addition, this study demonstrates no additional benefit to adding diastolic blood pressure, as has been incorporated into the CURB65 and CRB65 scores, over and above measurement of systolic blood pressure. A simplified score consisting of confusion, respiratory rate $\geqslant 30 / \mathrm{min}$, systolic blood pressure $<90 \mathrm{~mm} \mathrm{Hg}$ and age $\geqslant 65$ years has equivalent performance to the current CURB65 and CRB65 scores.
This study has shown that mean arterial pressure $<70 \mathrm{~mm} \mathrm{Hg}$, as used in the APACHE II score and widely in intensive care medicine, is a predictor of 30 day mortality and need for mechanical ventilation and/or inotropic support. Similarly reduced pulse pressure $\leqslant 40 \mathrm{~mm} \mathrm{Hg}$ is associated with increased 30 day mortality and need for mechanical ventilation and/or inotropic support. Experimental studies have shown an early increase in pulse pressure in sepsis, however, pulse pressure narrows later in sepsis. ${ }^{10}$ We hypothesised that if low diastolic blood pressure was a marker of severity independent of systolic blood pressure, then elevated pulse pressure would be a useful marker of outcome. In this study, however, an increased pulse pressure $>40 \mathrm{~mm} \mathrm{Hg}$ was not associated with increased 30 day mortality and need for mechanical ventilation and/or inotropic support. The admission systolic blood pressure alone had superior performance characteristics compared with both mean arterial pressure and pulse pressure.

Severity assessment is an increasingly important component of the management of community acquired pneumonia. Several scoring systems have been proposed for prediction of 30 day mortality in community acquired pneumonia over the past decade, all of which contain assessment of blood pressure..$^{2-6}$ Internationally, guidelines currently recommend the use of the Pneumonia Severity Index developed by Fine et al and/or the CURB65 score developed by $\mathrm{Lim}$ and colleagues. ${ }^{11}{ }^{13}$ The Pneumonia Severity Index was developed following a study in over 50000 patients and introduced in 1997. ${ }^{3}$ It is now well established worldwide and has been validated in large independent populations..$^{14}{ }^{15} \mathrm{It}$ is composed of 20 demographic, clinical, laboratory and radiographic variables. The British Thoracic Society independently developed a simpler scoring system (the CURB criteria) based on the "core adverse prognostic features" of confusion, urea $>7 \mathrm{mmol} / \mathrm{l}$, respiratory rate $\geqslant 30$ breaths $/ \mathrm{min}$ and systolic blood pressure $<90 \mathrm{~mm} \mathrm{Hg}$ and/or diastolic blood pressure $\leqslant 60 \mathrm{~mm} \mathrm{Hg} .{ }^{25}$ This was subsequently modified to the CURB65 score which is now widely used and has been independently validated in over 11000 patients in nine countries. ${ }^{16-21}$ The CRB65 score, which simplifies the score by omitting measurement of blood urea nitrogen, has been proposed for outpatient use and has been shown to have equivalent performance to CURB65 in some studies. ${ }^{20}$

All of these scoring systems contain assessment of admission blood pressure, and hypotension has been found to be associated with increased mortality in all of the major studies examining prognosis in patients with community acquired

Table 6 Modifications of the CURB65 and CRB65 scores for prediction of 30 day mortality and need for mechanical ventilation and/or inotropic support

\begin{tabular}{|c|c|c|c|c|c|c|}
\hline & $\begin{array}{l}\text { PPV } \\
(\%)\end{array}$ & $\begin{array}{l}\text { NPV } \\
(\%)\end{array}$ & $\begin{array}{l}\text { Sensitivity } \\
(\%)\end{array}$ & $\begin{array}{l}\text { Specificity } \\
(\%)\end{array}$ & AUC & p Value \\
\hline \multicolumn{7}{|l|}{ Prediction of 30 day mortality } \\
\hline CURB65 score & 20.9 & 95.7 & 70.1 & 80.4 & $0.76(0.74-0.79)$ & $<0.0001$ \\
\hline CUR65 + systolic $\mathrm{BP}<90 \mathrm{~mm} \mathrm{Hg}$ & 26.6 & 95.3 & 65.4 & 79.4 & $0.79(0.76-0.81)$ & $<0.0001$ \\
\hline CRB65 score & 28.6 & 94.0 & 47.4 & 87.4 & $0.74(0.71-0.77)$ & $<0.0001$ \\
\hline CR65 + systolic $\mathrm{BP}<90 \mathrm{~mm} \mathrm{Hg}$ & 36.8 & 93.6 & 40.2 & 92.6 & $0.76(0.73-0.79)$ & $<0.0001$ \\
\hline \multicolumn{7}{|c|}{ Prediction of need for mechanical ventilation and/or inotropic support } \\
\hline CURB65 score & 22.7 & 95.7 & 71.8 & 81.0 & $0.78(0.75-0.81)$ & $<0.0001$ \\
\hline CUR65 + systolic $\mathrm{BP}<90 \mathrm{~mm} \mathrm{Hg}$ & 25.1 & 95.0 & 63.1 & 79.7 & $0.79(0.76-0.81)$ & $<0.0001$ \\
\hline CRB65 score & 30.4 & 93.6 & 47.6 & 87.6 & $0.77(0.74-0.79)$ & $<0.0001$ \\
\hline CR65 + systolic BP $<90 \mathrm{~mm} \mathrm{Hg}$ & 36.8 & 92.9 & 37.9 & 92.6 & $0.77(0.74-0.80)$ & $<0.0001$ \\
\hline
\end{tabular}

AUC, area under the receiver operator characteristic curve; BP, blood pressure; NPV, negative predictive value; PPV, positive predictive value. 
pneumonia. The meta-analysis performed by Fine et al in 1996 identified systolic hypotension as one of the most powerful markers of poor outcome. ${ }^{4}$ The subsequent prediction rule developed by this group, the Pneumonia Severity Index, included systolic blood pressure as one of the core adverse features, with no assessment of diastolic blood pressure.

Although the Pneumonia Severity Index was found to be a robust clinical tool, its complexity made it difficult to implement into clinical practice. The advantage of the British Thoracic Society rules have therefore been their simplicity. Diastolic blood pressure $\leqslant 60 \mathrm{~mm} \mathrm{Hg}$ has been included in the British Thoracic Society rules since 1987 when a multicentre prospective study of 511 patients identified in multivariate logistic regression that diastolic hypotension was associated with increased mortality. ${ }^{2}$ Subsequent rules developed by the British Thoracic Society have all contained diastolic blood pressure $\leqslant 60 \mathrm{~mm} \mathrm{Hg}$ while also adding systolic blood pressure $<90 \mathrm{~mm} \mathrm{Hg}$.

In the study by Lim et al that developed the CURB65 score, however, $97 \%$ of patients with systolic hypotension ( $<90 \mathrm{~mm} \mathrm{Hg}$ ) also had diastolic hypotension ( $\leqslant 60 \mathrm{~mm} \mathrm{Hg}$ ) and it is not clear if including diastolic hypotension added any predictive value to the rule.

The accuracy of the CURB65 and CRB65 scores are now well established but they have not been universally accepted. Although simpler than the Pneumonia Severity Index, they are still sufficiently complex that a large majority of UK junior doctors were unable to name their components in one study. ${ }^{22}$ Despite increasing publicity and programmes to promote severity assessment, a recent study showed that only $13 \%$ of patients received severity assessment on admission. ${ }^{23}$ Some have suggested that pneumonia specific tools should be supplanted by generic illness tools, as the abundance of severity assessment scores for different conditions may confuse junior doctors. ${ }^{24}$ Investigators continue to develop new prediction rules and it is of note that systolic blood pressure alone has been used in these subsequent rules. ${ }^{25} 26$

If increasing simplicity is required, could the CURB65 be simplified by removing the diastolic blood pressure component? The results of this study show that doing so does not reduce the performance of the score for either 30 day mortality or the need for mechanical ventilation and/or inotropic support. The CURB65 and CRB65 scores had equivalent performance for both outcomes in this study. The CURB65 score can therefore be simplified to a modified CRB65 score by excluding the diastolic blood pressure component without compromising its accuracy. This study included only patients assessed in hospital with community acquired pneumonia. The CRB65 score has also been proposed for severity assessment in outpatients with community acquired pneumonia and although it is likely that the findings of this study would be true for outpatients as well, this has not been studied.

In conclusion, admission systolic blood pressure can predict both 30 day mortality and the need for mechanical ventilation and/or inotropic support. Systolic blood pressure is superior to other haemodynamic predictors of severity in community acquired pneumonia. The CURB65 score can be simplified to a modified CRB65 score by excluding the diastolic component of blood pressure assessment without compromising its accuracy. Independent validation of this modified score is needed.

Competing interests: None.

Ethics approval: The study was approved by the Lothian Research Ethics Committee.

\section{REFERENCES}

1. Rivers $\mathbf{E}$, Nguyen B, Havstad $\mathrm{S}$, et al. Early goal-directed therapy in the treatment of severe sepsis and septic shock. N Engl J Med 2001;345:1368-77.

2. British Thoracic Society and the Public Health Laboratory Service Community-acquired pneumonia in adults in UK hospitals in 1982-1983: a survey of aetiology, mortality, prognostic factors and outcome. O J Med 1987;62:195-220.

3. Fine MJ, Auble TE, Yealy DM, et al. A prediction rule to identify low-risk patients with community-acquired pneumonia. N Engl J Med 1997;336:243-50.

4. Fine MJ, Smith MA, Carson CA, et al. Prognosis and outcomes of patients with community-acquired pneumonia. A meta-analysis. JAMA 1996;275:134-41.

5. Neill AM, Martin IR, Weir R, et al. Community acquired pneumonia: aetiology and usefulness of severity criteria on admission. Thorax 1996;51:1010-16.

6. Lim WS, van der Eerden MM, Laing $R$, et al. Defining community acquired pneumonia severity on presentation to hospital: an international derivation and validation study. Thorax 2003;58:377-82.

7. Guidelines for the Management of Community Acquired Pneumona in Adults 2004 update. London: British Thoracic Society, 2004.

8. Knaus WA, Draper EA, Wagner DP, et al. Apache II: A severity of disease classification system. Crit Care Med 1985;13:818-29.

9. Subbe $\mathbf{C P}$, Kruger $\mathrm{M}$, Rutherford $\mathrm{P}$, et al. Validation of a modified Early Warning Score in medical admissions. Q J Med 2001;94:521-6.

10. Sam AD II, Sharma AC, Law WR, et al. Splanchic vascular control during sepsis and endotoxemia. Front Biosci 1997;1;2:e72-92.

11. British Thoracic Society Standards of Care Committee. BTS guidelines for the management of community acquired pneumonia in adults. Thorax 2001;56/Suppl IV):iv1-64.

12. Swets J. A Measuring the accuracy of diagnostic systems. Science 1998;3;240:1285-93.

13. Mandell LA, Wunderink RG, Anzueto A, et al. Infectious Disease Society of American/American Thoracic Society Consensus Guidelines for the Management of Community Acquired Pneumonia in Adults. Clin Infect Dis 2007;44:S27-72.

14. España PP, Capelastegui A, Quintana JM, et al. A prediction rule to identify allocation of inpatient care in community-acquired pneumonia. Eur Respir $J$ 2003;21:695-701.

15. Marras TK, Gutierrez C, Chan CK. Applying a prediction rule to identify low-risk patients with community-acquired pneumonia. Chest 2000;118:1339-43.

16. Man SY, Lee N, Ip M, et al. Prospective comparison of three predictive rules for assessing severity of community-acquired pneumonia in Hong Kong. Thorax 2007:62:348-53.

17. Aujesky D, Auble TE, Yealy DM, et al. Prospective comparison of three validated prediction rules for prognosis in community-acquired pneumonia. Am J Med 2005;118:384-92.

18. Buising $\mathbf{K L}$, Thursky KA, Black JF, et al. A prospective comparison of severity scores for identifying patients with severe community acquired pneumonia: reconsidering what is meant by severe pneumonia. Thorax 2006;61:419-24.

19. Spindler C, Ortqvist A. Prognostic score systems and community-acquired bacteraemic pneumococcal pneumonia. Eur Respir J 2006;28:816-23.

20. Capelastegui A, Espana PP, Quintana JM, et al. Validation of a predictive rule for the management of community-acquired pneumonia. Eur Respir J 2006;27:151-7.

21. Barlow GD, Nathwani D, Davey PG. The CURB65 pneumonia severity score outperforms generic sepsis and early warning scores in predicting mortality in community-acquired pneumonia. Thorax 2007:62:253-9.

22. Barlow G, Nathwani D, Myers $\mathrm{E}$, et al. Identifying barriers to the rapid administration of appropriate antibiotics in community-acquired pneumonia. J Antimicrob Chemother 2008:61:442-51.

23. Collini $\mathbf{P}$, Beadsworth $\mathrm{M}$, Anson J. Community-acquired pneumonia: doctors do not follow national guidelines. Postgrad Med J 2007;83:552-5.

24. Woodhead M. Assessment of illness severity in community acquired pneumonia: a useful new prediction tool? Thorax 2003:58:371-2.

25. Espana PP, Capelastegui A, Gorordo I, et al. Development and validation of a clinical prediction rule for severe community-acquired pneumonia. Am J Respir Crit Care Med 2006;174:1249-56.

26. Myint PK, Kamath AV, Vowler SL, et al. Severity assessment criteria recommended by the British Thoracic Society (BTS) for community-acquired pneumonia (CAP) and older patients. Should SOAR (systolic blood pressure, oxygenation, age and respiratory rate) criteria be used in older people? A compilation study of two prospective cohorts. Age Ageing 2006;35:286-91. 